\title{
On Finite Mixtures of Modified Intervened Poisson Distribution And Its Applications
}

\author{
C. Satheesh Kumar \\ Department of Statistics, University of Kerala \\ Trivandrum-695581, Kerala, India. \\ drcsatheeshkumar@gmail.com \\ D.S. Shibu \\ Department of Statistics, University College \\ Trivandrum-695034, Kerala, India. \\ dsshibu70@ymail.com \\ Received 2 February 2013 \\ Accepted 11 July 2014
}

\begin{abstract}
Kumar and Shibu proposed a modified version of intervened Poisson distribution (IPD), namely the modified intervened Poisson distribution (MIPD) for tackling situations of further interventions useful for certain practical problems. Here we consider some finite mixtures of MIPD and study some of its important properties. The identifiability condition of the mixture distribution is derived and the parameters of the mixture model are estimated by various methods such as method of factorial moments and method of maximum likelihood. In addition, this mixture model is fitted to some real life data sets.
\end{abstract}

Key words: Method of factorial moments; mixture distribution; probability generating function.

AMS (2000) Subject Classification: Primary 60E05, 60E10

\section{Introduction}

Finite mixtures of distributions have provided a mathematical based approach to the statistical modeling of a wide variety of random phenomena. Because of their usefulness as an extremely flexible method of modeling, finite mixture models have an increasing attention over the years from practical and theoretical point of view. Indeed, in the past decade the extent and the potential of the application of finite mixture models have widened considerably. Application of mixture models spread over astronomy, biology, genetics, medicine, psychiatry, economics, engineering, marketing and other fields in the biological, physical and social sciences. For details see McLachlan and Peel [7]. In these applications, finite mixture 
models support a variety of techniques in major areas of statistics including cluster and latent class analysis, discriminant analysis, image analysis and survival analysis. There is a vast literature available on finite mixture models. For example, see Everitt and Hand [2], Titterington et al. [12] and the review article of Titterington [11].

Cohen [1] introduced zero truncated Poisson distribution (ZTPD) to describe a chance mechanism whose observational apparatus becomes active only when at least one event occurs. Singh [10] considered a numerical example to illustrate the statistical application of the ZTPD in such situations. Later, a modified version of ZTPD is introduced by Shanmugam [8] which he termed as the intervened Poisson distribution (IPD). The IPD has been found applications in several areas of research such as reliability analysis, queuing problems, epidemiological studies etc. For example, see Huang and Fung [3] and Shanmugam $[8,9]$. During the observational period, the failed units are either replaced by new units or rebuilt. This kind of replacement changes the reliability of a system as only some of its components have longer life. Kumar and Shibu [5] modified the IPD in order to incorporate the situation of further intervention and Kumar and Shibu [6] obtained alternative form of the truncated IPD.

Here, we develop certain finite mixtures of the modified IPDs (MIPDs). This mixture model can address the situations of further interventions. The paper is organized as follows. In section 2, we present some important aspects of the IPD and MIPD and in section 3, we consider some finite mixtures of

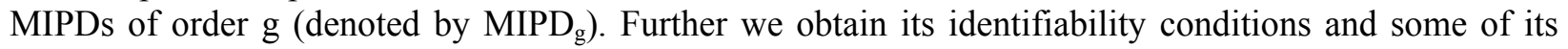
important properties in that section. Estimation of the parameters of MIPD $_{\mathrm{g}}$ by method of factorial moments and method of maximum likelihood are discussed in section 4 and illustrated using certain real life data sets.

\section{The intervened Poisson distribution and its modified form}

Shanmugam [8] developed the intervened Poisson distribution as in the following:

He assumed that there is an intervention during the observational period and its effect is to change the Poisson parameter $\lambda$ to $\rho \lambda$. Let $U=U_{1}+U_{2}$. $U_{1}$ is the number of cases before intervention and $U_{2}$ is the number of cases after intervention. The observational apparatus is activated only when $U_{1}>0$. Hence, $U_{1}$ is assumed to be a zero truncated Poisson variate with parameter $\lambda$, and $U_{2}$ is an independent Poisson variate with parameter $\rho \lambda$. Shanmugam [8] obtained the distribution of $U$ and its statistical properties, and gave an example to illustrate its application. The probability mass function ( pmf) of the $\operatorname{IPD}(\lambda, \rho)$ is given by

$$
\begin{aligned}
g(u)=P & (U=u) \\
= & \frac{\left[(1+\rho)^{u}-\rho^{u}\right]}{\left(e^{\lambda}-1\right) e^{\lambda \rho}} \frac{\lambda^{u}}{u !},
\end{aligned}
$$

with $\lambda>0$ and $\rho \geq 0$ for those values of $u$ on the positive integers, and zero elsewhere.

The mean and variance of the $I P D$ with $\mathrm{pmf}(2.1)$ are

Published by Atlantis Press

Copyright: the authors 


$$
E(U)=\lambda(\rho+q)=\mu
$$

and

$$
\operatorname{Var}(U)=\mu-\lambda^{2} q(q-1)
$$

where

$$
q=e^{\lambda}\left(e^{\lambda}-1\right)^{-1}
$$

From (2.3) and (2.4), it can be observed that the $\operatorname{IPD}(\lambda, \rho)$ is under dispersed (that is, its variance is less than its mean), for all values of the parameters.

Now, we discuss the derivation of the modified version of intervened Poisson distribution (MIPD) due to Kumar and Shibu [5].

Let $V_{1}$ be a positive integer valued random variable following the IPD with parameters $\lambda$ and $\rho_{1}$, and let $V_{2}$ be a non-negative integer valued random variable having Poisson distribution with mean $\lambda \rho_{2}$, in which $\lambda>0$ and $\rho_{j} \geq 0$ for each $j=1,2$. Assume that $V_{1}$ and $V_{2}$ are statistically independent. Then the distribution of $V=V_{1}+2 V_{2}$ is called the modified intervened Poisson distribution with parameters $\lambda, \rho_{1}$ and $\rho_{2}$ which we written as $\operatorname{MIPD}\left(\lambda, \rho_{1}, \rho_{2}\right)$. Clearly $\operatorname{MIPD}(\lambda, \rho, 0)$ is $\operatorname{IPD}(\lambda, \rho)$. Thus the $\operatorname{MIPD}\left(\lambda, \rho_{1}, \rho_{2}\right)$ is an extended class of discrete distributions which include both truncated Poisson distribution and the $\operatorname{IPD}(\lambda, \rho)$ as its special case. Also, this type of an extension opens up the possibility of a second intervention. Now we present some important properties of the $\operatorname{MIPD}\left(\lambda, \rho_{1}, \rho_{2}\right)$.

Result 2.1. The probability mass function $g_{x}=P(X=x)$ of $\operatorname{MIPD}\left(\lambda, \rho_{1}, \rho_{2}\right)$ is the following for $x=1,2, \ldots$ in which $\lambda>0$ and $\rho_{j} \geq 0$ for each $j=1,2$.

$$
g_{x}=c \sum_{r=0}^{\left[\frac{x-1}{2}\right]} \frac{\delta_{x-2 r}\left(\lambda \rho_{2}\right)^{r}}{(x-2 r) ! r !}
$$

where [a] denotes the integral part of a,

$$
\delta_{x}=\lambda^{x}\left[(1+\rho)^{x}-\rho^{x}\right]
$$

for $x \geq 0$ and 
Kumar and Shibu

$$
c=e^{-\lambda\left(\rho_{1}+\rho_{2}\right)}\left(e^{\lambda}-1\right)^{-1}
$$

Result 2.2 The Probability generating function (pgf) of the $\operatorname{MIPD}\left(\lambda, \rho_{1}, \rho_{2}\right)$ with pmf (2.5) is the following.

$$
Q(s)=c\left(e^{\lambda s}-1\right) e^{\lambda s\left(\rho_{1}+\rho_{2} s\right)}
$$

Result 2.3 The mean and variance of the $\operatorname{MIPD}\left(\lambda, \rho_{1}, \rho_{2}\right)$ are the following.

$$
E(V)=\lambda\left[q+\rho_{1}+\rho_{2}\right]
$$

and

$$
\operatorname{Var}(V)=\lambda\left[\rho_{1}+q-q(q-1)+4 \rho_{2}\right]
$$

Result 2.4 The r-th factorial moment $\mu_{[r]}$ of $\operatorname{MIPD}\left(\lambda, \rho_{1}, \rho_{2}\right)$ with pgf (2.8) is the following, for $r=1,2, \ldots$

$$
\mu_{[r]}=\frac{r !}{\left(e^{\lambda}-1\right)} \sum_{j=0}^{\left[\frac{r}{2}\right]} \frac{\left(\lambda \rho_{2}\right)^{j} \lambda^{r-2 j}}{(r-2 j) !}\left[e^{\lambda}\left(1+\rho+2 \rho_{2}\right)^{r-2 j}-\left(\rho_{1}+2 \rho_{2}\right)^{r-2 j}\right]
$$

Remark 2.1 If we put $\rho_{2}=0, \rho_{1}=\rho$ in (2.11), then we get the rth factorial moment of $\operatorname{IPD}(\lambda, \rho)$ as

$$
\mu_{[r]}=\lambda^{r}\left(e^{\lambda}-1\right)^{-1}\left[e^{\lambda}(1+\rho)^{r}-\rho^{r}\right] .
$$

Result 2.5 A simple recurrence relation for the probabilities of $\operatorname{MIPD}\left(\lambda, \rho_{1}, \rho_{2}\right)$ is the following, for $x=1,2, .$.

$$
(x+1) g_{x+1}=\lambda\left(1+\rho_{1}\right) g_{x}+2 \lambda \rho_{2} g_{x-1}+c \lambda \sum_{j=0}^{\left[\frac{x}{2}\right]} \frac{\left(\lambda \rho_{1}\right)^{x-2 j}\left(\lambda \rho_{2}\right)^{j}}{(x-2 j) ! j !}
$$

Result 2.6 Recurrence relation for the factorial moments $\mu_{[r]}$ of $\operatorname{MIPD}\left(\lambda, \rho_{1}, \rho_{2}\right)$ is the following, 
for $r \geq 1$, in which $\Delta_{j}=\sum_{j=0}^{\left[\frac{r}{2}\right]} \frac{\left[\lambda\left(\rho_{1}+2 \rho_{2}\right)\right]^{r-2 j}\left(\lambda \rho_{2}\right)^{j}}{(r-2 j) ! j !}, \mu_{[0]}=1$ and $\mu_{[-r]}=0$ for $r>0$.

$$
\mu[r+1]=\lambda\left[1+\rho_{1}+2 \rho_{2}\right] \mu_{[r]}+2 \lambda \rho_{2} r \mu_{[r-1]}+r ! \lambda\left(e^{\lambda}-1\right)^{-1} \Delta_{j}
$$

\section{Mixtures of modified intervened Poisson distribution}

In this section, first we present some general idea about mixture distribution. Let $X$ be a discrete random variable with pmf $p(x)=P(X=x)$ of the form $p(x)=\alpha_{1} p_{1}(x)+\alpha_{2} p_{2}(x)+\ldots+\alpha_{g} p_{g}(x)$ where for each $j=1,2, \ldots, g, \alpha_{j}>0$ such that $\sum_{j=1}^{g} \alpha_{g}=1$ and $p_{j}(x) \geq 0$ such that $\sum_{x} p_{j}(x)=1$. Then we say that $X$ has a mixture distribution and $p(x)$ is a finite mixture distribution. The parameters $\alpha_{1}, \alpha_{2}, \ldots, \alpha_{g}$ are known as mixing weights and $p_{1}, p_{2}, \ldots, p_{g}$, the components of the mixture. We denote $\Theta$ as the collection of all distinct parameters occurring in the components and $\psi$ as the complete collection of all distinct parameters occurring in the mixture model.

Let $\Delta=\left\{F\left(x, \theta_{j}\right): \theta_{j} \in \Theta, x \in R\right\}$ be the class of distribution functions from which mixtures are to be formed. We identify the class of finite mixtures of $\Delta$ with the appropriate class of distribution functions, defined by $\hat{H}=\left\{H(x): H(x)=\sum_{j=1}^{g} \alpha_{j} F\left(x, \theta_{j}\right), \alpha_{j}>0, F\left(; \theta_{j}\right) \in \Delta, j=1,2, \ldots, g\right\}$ so that $\hat{H}$ is the convex hull of $\Delta$, we denote $F\left(x, \theta_{j}\right)$ by $F_{j}(x)$ or simply $F_{j}$ and the mixture by $H=\sum_{j=1}^{g} \alpha_{j} F_{j}$.

We need the following theorem from Titteringtonet.al. (1985) in order to establish the identifiability condition of the mixture models considered in this paper.

Theorem 3.1 (Titterington et.al. [12]) A necessary and sufficient condition for $\hat{H}$ to be identifiable is that $\Delta$ is linearly independent over the field of real numbers.

Now, we present the definition and some important properties of finite mixtures of $\operatorname{MIPD}\left(\lambda, \rho_{1}, \rho_{2}\right)$.

Definition 3.1 A random variable $Y$ is said to have a $g$ component mixture of MIPDs if it has the following $\operatorname{pmf} f(x)=P(Y=x)$, in which $0 \leq \alpha_{i} \leq 1$ for $i=1,2, \ldots, g$ with $\sum_{i=1}^{g} \alpha_{i}=1$ and $x=1,2, \ldots$

$$
f(x)=\sum_{i=1}^{g} \alpha_{i} f_{i}(x)
$$

where 


$$
\begin{gathered}
f_{i}(x)=k_{i} \sum_{r=0}^{\left[\frac{x-1}{2}\right]} \frac{\eta_{i}(x-2 r)\left(\lambda_{i} \mu_{i}\right)^{r}}{(x-2 r) ! r !} \\
k_{i}=e^{-\lambda_{i}\left(\rho_{i}+\mu_{i}\right)\left(e^{\lambda_{i}}-1\right)^{-1}}
\end{gathered}
$$

and

$$
\eta_{i}(x)=\lambda_{i}^{x}\left[\left(1+\rho_{i}\right)^{x}-\rho_{i}^{x}\right]
$$

with $\lambda_{i}>0, \rho_{i} \geq 0$ and $\mu_{i} \geq 0$ for each $i=1,2, \ldots, g$. Here after, we denote the distribution with pmf (3.2) by $M M I P D_{g}$. Now we present the identifiability condition of the $M M I P D_{g}$ through the following proposition.

\section{Result 3.1}

The identifiability condition for MMIPD $D_{g}$ with $\operatorname{pmf} f(x)$ given in (3.1) is $\lambda_{i} \neq \lambda_{j}, \rho_{i} \neq \rho_{j}$ and $\mu_{i} \neq \mu_{j}$ for $i \neq j$ taking values from $1,2, \ldots, g$ and $x=1,2, \ldots$

Proof Assuming $g=2$ and consider the equation

$$
b_{1} F_{1}(x)+b_{2} F_{2}(x)=0
$$

where $b_{1}$ and $b_{2}$ are any two arbitrary real numbers, $F_{1}(x)=\sum_{j=1}^{x} f(j)$ and $F_{2}(x)=\sum_{j=1}^{x} \phi(j)$ for $x=1,2, \ldots$ in which $\phi(j)$ obtained from $f(j)$ by replacing $\lambda_{j}$ by $\beta_{j}, \rho_{j}$ by $\theta_{j}$ and $\mu_{j}$ by $\gamma_{j}$. Assume that for each $i=1,2, \lambda_{i} \neq \beta_{i}, \quad \rho_{i} \neq \theta_{i}$ and $\mu_{i} \neq \gamma_{i}$. Thus for $\alpha_{1}=\alpha$, we have

$$
\begin{gathered}
F_{1}(x)=\frac{1}{j !} \sum_{j=1}^{x}\left[\alpha \sum_{r=0}^{\left[\frac{j-1}{2}\right]} \frac{k_{1} \eta_{1}(j-2 r)\left(\lambda_{1} \mu_{1}\right)^{r}}{(j-2 r) ! r !}+(1-\alpha) \sum_{r=0}^{\left[\frac{j-1}{2}\right]} \frac{k_{2} \eta_{2}(j-2 r)\left(\lambda_{2} \mu_{2}\right)^{r}}{(j-2 r) ! r !}\right] \\
F_{2}(x)=\frac{1}{j !} \sum_{j=1}^{x}\left[\alpha\left[\frac{j-1}{2}\right] \sum_{r=0}^{\frac{c_{1}}{(j-2 r) ! r !}+(j-2 r)\left(\beta_{1} \gamma_{1}\right)^{r}}+(1-\alpha) \sum_{r=0}^{\frac{j-1}{2}} \frac{\xi_{2}}{(j-2 r) ! r !}\right]
\end{gathered}
$$

Now from equations (3.5), (3.6) and (3.7), we have the following: 


$$
\begin{aligned}
& \left.\sum_{j=1}^{x} \sum_{r=0}^{\left[\frac{j-1}{2}\right]}\right]\left[\frac{b_{1} k_{1} \eta_{1}(j-2 r)\left(\lambda_{1} \mu_{1}\right)^{r}+b_{2} c_{1} \xi_{1}(j-2 r)\left(\beta_{1} \gamma_{1}\right)^{r}}{(j-2 r) ! r !}\right]=0 \\
& \sum_{j=1}^{x} \sum_{r=0}^{\left[\frac{j-1}{2}\right]}\left[\frac{b_{1} k_{1} \eta_{1}(j-2 r)\left(\lambda_{1} \mu_{1}\right)^{r}+b_{2} c_{1} \xi_{1}(j-2 r)\left(\beta_{1} \gamma_{1}\right)^{r}}{(j-2 r) ! r !}\right]=0
\end{aligned}
$$

Equations (3.8) and (3.9) yield the following.

$$
b_{1}\left[\sum_{j=1}^{x} \sum_{r=0}^{\left[\frac{j-1}{2}\right]} \frac{\left\{k_{1} \eta_{1}(j-2 r)\left(\lambda_{1} \rho_{1}\right)^{r} c_{2} \xi_{2}(j-2 r)\left(\beta_{2} \gamma_{2}\right)^{r}\right\}}{(j-2 r) ! r !}\right]=b_{1}\left[\sum_{j=1}^{x} \sum_{r=0}^{\left[\frac{j-1}{2}\right]} \frac{\left\{k_{2} \eta_{2}(j-2 r)\left(\lambda_{2} \mu_{2}\right)^{r} c_{1} \xi_{1}(j-2 r)\left(\beta_{1} \gamma_{1}\right)^{r}\right\}}{(j-2 r) ! r !}\right] \text {, }
$$

which implies $b_{1}=0$ and hence $b_{2}=0$ by (3.5). Hence it can be concluded by theorem 1.1 that $F_{1}$ and $F_{2}$ are linearly independent. Now, the argument can be extended to case of any positive integer $g$ and thus the proof follows.

Result 3.2 The mean and variance of $M M I P D_{g}$ with pmf $f(x)$ given in (3.1) are the following.

$$
E(Y)=\sum_{i=1}^{g} \alpha_{i} \lambda_{i}\left(q_{i}+\rho_{i}+2 \mu_{i}\right)=\sum_{i=1}^{g} \tau_{i}=\tau
$$

where for $i=1,2, \ldots, g$

$$
\begin{gathered}
q_{i}=e^{\lambda_{i}}\left(e^{\lambda_{i}}-1\right)^{-1} \\
\operatorname{Var}(Y)=\sum_{i=1}^{g} \alpha_{i}\left[\lambda_{i}\left\{\rho_{i}+q_{i}-\lambda_{i} q_{i}\left(q_{i}-1\right)+4 \mu_{i}\right\}+\lambda_{i}^{2}\left\{\rho_{i}+q_{i}+2 \mu_{i}\right\}^{2}\right]-\tau^{2}
\end{gathered}
$$

The proof is simple and hence omitted.

Result 3.3 The probability generating function (pgf) of $M M I P D_{g}$ with pmf (3.1) is the following.

$$
P(s)=\sum_{i=1}^{g} \alpha_{i} k_{i}\left(e^{\lambda_{i} s}-1\right) e^{\lambda_{i} s\left(\rho_{i}+\mu_{i} s\right)}
$$


Proof follows from the definition of the pgf and equations given in (2.8) and (3.1).

\section{Result 3.4}

The rth factorial moment $v_{[r]}$ of $M M I P D_{g}$ with pmf $f(x)$ given in (3.1) is the following for $r=1,2, \ldots$

$$
v_{[r]}=\sum_{i=1}^{g} \sum_{j=0}^{\left[\frac{r}{2}\right]} \frac{\alpha_{i}\left(\lambda_{i} \mu_{i}\right)^{j} \lambda_{i}^{r-2 j}}{\left(e^{\lambda_{i}}-1\right) j !(r-2 j) !}\left[e^{\lambda_{i}}\left\{1+\rho_{i}+2 \mu_{i}\right\}^{r-2 j}-\left\{\rho_{i}+2 \mu_{i}\right\}^{r-2 j}\right]
$$

Proof The $r$ th factorial moment $v_{[r]}$ of $M M I P D_{g}$ is

$$
\begin{gathered}
v_{[r]}=E[Y(Y-1) \ldots(Y-r+1)] \\
=\sum_{x=r}^{\infty} \frac{x !}{(x-r) !} f(x) .
\end{gathered}
$$

Now the proof follows from (2.11) and (3.1).

\section{Estimation}

In this section we discuss the estimation of the parameters of the mixture model $M M I P D_{2}$ by method of factorial moments and method of maximum likelihood. In the method of factorial moment estimation, the first seven population factorial moments of $M M I P D_{2}$ are equated to the corresponding sample factorial moments and obtain the following system of equations:

$$
v_{[r]}=\tau_{[r]}
$$

In method of maximum likelihood estimation, the parameters of the mixture models are estimated by maximizing the following log likelihood function with respect to the parameters.

$$
\log L=\sum_{x=1}^{z} n_{x} \log f(x)
$$

where $\mathrm{f}(\mathrm{x})$ is the probability model of the mixture, $n_{x}$ is the observed frequency of $x$ events and $z$ is the highest value of $x$ observed. Thus the maximum likelihood estimates of the parameters of the $M M I P D_{2}$ are obtained by solving the following system of equations in which for $i=1,2$,

$$
\Lambda_{i}=e^{\lambda_{i}}\left(1+\rho_{i}+\mu_{i}\right)-\left(\rho_{i}+\mu_{i}\right)
$$




$$
\begin{aligned}
& \alpha k_{1} \sum_{x=1}^{\infty} \sum_{r=0}^{\left[\frac{x-1}{2}\right]} \frac{n_{x} \eta_{1}(x-2 r)\left(\lambda_{1} \mu_{1}\right)^{r}}{f(x)(x-2 r) ! r !}=\alpha k_{2} \sum_{x=1}^{\infty} \sum_{r=0}^{\left[\frac{x-1}{2}\right]} \frac{n_{x} \eta_{2}(x-2 r)\left(\lambda_{2} \mu_{2}\right)^{r}}{f(x)(x-2 r) ! r !} \\
& \alpha \sum_{x=1}^{\infty} \sum_{r=0}^{\left[\frac{x-1}{2}\right]} \frac{(x-r) n_{x} \eta_{1}(x-2 r)\left(\lambda_{1} \mu_{1}\right)^{r}}{f(x)(x-2 r) ! r !}=\alpha \lambda_{1} \sum_{x=1}^{\infty} \sum_{r=0}^{\left[\frac{x-1}{2}\right]} \frac{n_{x} \eta_{1}(x-2 r)\left(\lambda_{1} \mu_{1}\right)^{r}}{f(x)(x-2 r) ! r !} \Lambda_{1} \\
& \alpha \sum_{x=1}^{\infty} \sum_{r=0}^{\left[\frac{x-1}{2}\right]} \frac{(x-r) n_{x} \eta_{1}(x-2 r)\left(\lambda_{2} \mu_{2}\right)^{r}}{f(x)(x-2 r) ! r !}=\alpha \lambda_{1} \sum_{x=1}^{\infty} \sum_{r=0}^{\left[\frac{x-1}{2}\right]} \frac{n_{x} \eta_{1}(x-2 r)\left(\lambda_{2} \mu_{2}\right)^{r}}{f(x)(x-2 r) ! r !} \Lambda_{2} \\
& \alpha \sum_{x=1}^{\infty} \sum_{r=0}^{\left[\frac{x-1}{2}\right]} \frac{n_{x} \eta_{1}(x-2 r-1)\left(\lambda_{1} \mu_{1}\right)^{r}}{f(x)(x-2 r-1) ! r !}=\alpha \sum_{x=1}^{\infty} \sum_{r=0}^{\left[\frac{x-1}{2}\right]} \frac{n_{x} \eta_{1}(x-2 r)\left(\lambda_{1} \mu_{1}\right)^{r}}{f(x)(x-2 r) ! r !} \\
& \alpha \sum_{x=1}^{\infty} \sum_{r=0}^{\left[\frac{x-1}{2}\right]} \frac{n_{x} \eta_{1}(x-2 r-1)\left(\lambda_{2} \mu_{2}\right)^{r}}{f(x)(x-2 r-1) ! r !}=\alpha \sum_{x=1}^{\infty} \sum_{r=0}^{\left[\frac{x-1}{2}\right]} \frac{n_{x} \eta_{1}(x-2 r)\left(\lambda_{2} \mu_{2}\right)^{r}}{f(x)(x-2 r) ! r !} \\
& \alpha \sum_{x=1}^{\infty} \sum_{r=0}^{\left[\frac{x-1}{2}\right]} \frac{n_{x} \eta_{1}(x-2 r)\left(\lambda_{1} \mu_{1}\right)^{r}}{f(x)(x-2 r) ! r !}=\alpha \sum_{x=1}^{\infty} \sum_{r=0}^{\left[\frac{x-1}{2}\right]} \frac{n_{x} \eta_{1}(x-2 r)\left(\lambda_{1} \mu_{1}\right)^{r+1}}{f(x)(x-2 r) ! r !} \\
& \alpha \sum_{x=1}^{\infty} \sum_{r=0}^{\left[\frac{x-1}{2}\right]} \frac{n_{x} \eta_{1}(x-2 r)\left(\lambda_{2} \mu_{2}\right)^{r}}{f(x)(x-2 r) ! r !}=\alpha \sum_{x=1}^{\infty} \sum_{r=0}^{\left[\frac{x-1}{2}\right]} \frac{n_{x} \eta_{1}(x-2 r)\left(\lambda_{2} \mu_{2}\right)^{r+1}}{f(x)(x-2 r) ! r !}
\end{aligned}
$$

We consider the fitting of $M M I P D_{2}$ to real life data sets by the method of factorial moments and method of maximum likelihood and presented in Tables 1 and 2 . The dataset given in table 1 indicates the distribution of number of articles on theoretical Statistics and Probability for years 1940-49 and initial letter N-R of the author's name. For reference, see [4]. The second data set given in table 2 represents the distribution of 1534 biologists according to the number of research papers to their credit in the review of applied entomology, volume 24, 1936. For details, see [13]. Also, we compare the estimates of parameters of $M_{M I P D_{2}}$ with two component mixture of intervened Poisson distribution (TMIPD) and two component mixture of truncated Poisson distribution (TMTPD). From Tables 1 and 2, it is seen that $M M I P D_{2}$ gives the best fit over TMIPD and TMTPD. For the data-set in Table 1 , we assume that $\lambda_{1}=\lambda_{2}$ and $\rho_{1}=\rho_{2}$. 
Table 1. Comparison of fit of $M M I P D_{2}$ using various methods of estimation for the first data-set

\begin{tabular}{|c|c|c|c|c|c|c|c|}
\hline \multirow{2}{*}{ count } & \multirow{2}{*}{$\begin{array}{c}\text { Observed } \\
\text { 'f' }\end{array}$} & \multicolumn{3}{|c|}{ Expected frequency by factorial moments } & \multicolumn{3}{|c|}{ Expected frequency by maximum likelihood } \\
\hline & & MZTPD & MIPD & $\mathrm{MMIPD}_{2}$ & MZTPD & MIPD & $\mathrm{MMPD}_{2}$ \\
\hline 1 & 83 & 71 & 69 & 75 & 71 & 73 & 78 \\
\hline 2 & 18 & 24 & 20 & 22 & 21 & 22 & 20 \\
\hline 3 & 13 & 20 & 18 & 16 & 18 & 17 & 15 \\
\hline 4 & 9 & 10 & 10 & 10 & 12 & 13 & 13 \\
\hline 5 & 7 & 8 & 10 & 10 & 10 & 6 & 6 \\
\hline 6 & 7 & 6 & 6 & 6 & 6 & 6 & 6 \\
\hline 7 & 2 & 2 & 2 & 2 & 2 & 2 & 2 \\
\hline 8 & 5 & 3 & 3 & 3 & 4 & 5 & 5 \\
\hline Total & 144 & 144 & 144 & 144 & 144 & 144 & 144 \\
\hline \multicolumn{2}{|c|}{$\begin{array}{l}\text { Degrees of } \\
\text { freedom }\end{array}$} & 4 & 3 & 1 & 4 & 3 & 1 \\
\hline \multicolumn{2}{|c|}{$\begin{array}{l}\text { Estimated values } \\
\text { of parameters }\end{array}$} & $\begin{array}{l}\hat{\alpha}=0.28 \\
\hat{\lambda}_{1}=0.64 \\
\hat{\lambda}_{2}=0.21\end{array}$ & $\begin{array}{l}\hat{\alpha}=0.28 \\
\hat{\lambda}_{1}=0.59 \\
\hat{\lambda}_{2}=0.25 \\
\hat{\rho}_{1}=0.78 \\
\hat{\rho}_{2}=0.41\end{array}$ & $\begin{array}{l}\hat{\alpha}=0.67 \\
\hat{\lambda}_{1}=0.28 \\
\hat{\lambda}_{2}=0.23 \\
\hat{\rho}_{1}=0.39 \\
\hat{\rho}_{2}=0.52 \\
\hat{\mu}_{1}=0.19 \\
\hat{\mu}_{1}=0.43\end{array}$ & $\begin{array}{l}\hat{\alpha}=0.35 \\
\hat{\lambda}_{1}=0.69 \\
\hat{\lambda}_{2}=0.19\end{array}$ & $\begin{array}{l}\hat{\alpha}=0.36 \\
\hat{\lambda}_{1}=0.44 \\
\hat{\lambda}_{2}=0.32 \\
\hat{\rho}_{1}=0.59 \\
\hat{\rho}_{2}=0.49\end{array}$ & $\begin{array}{l}\hat{\alpha}=0.46 \\
\hat{\lambda}_{1}=0.33 \\
\hat{\lambda}_{2}=0.29 \\
\hat{\rho}_{1}=0.45 \\
\hat{\rho}_{2}=0.56 \\
\hat{\mu}_{1}=0.22 \\
\hat{\mu}_{1}=0.35\end{array}$ \\
\hline \multicolumn{2}{|c|}{ Chi-square value } & 7.17 & 6.40 & 4.11 & 5.83 & 4.60 & 2.35 \\
\hline \multicolumn{2}{|c|}{ P-value } & 0.31 & 0.38 & 0.66 & 0.44 & 0.60 & 0.89 \\
\hline
\end{tabular}


Table 2. Comparison of fit of $M M I P D_{2}$ using various methods of estimation for the second data-set

\begin{tabular}{|c|c|c|c|c|c|c|c|}
\hline \multirow{2}{*}{ count } & \multirow{2}{*}{$\begin{array}{l}\text { Observed } \\
\text { 'f' }\end{array}$} & \multicolumn{3}{|c|}{ Expected frequency by factorial moments } & \multicolumn{3}{|c|}{ Expected frequency by maximum likelihood } \\
\hline & & MZTPD & MIPD & $\mathrm{MMIPD}_{2}$ & MZTPD & MIPD & $\mathrm{MMPD}_{2}$ \\
\hline 1 & 365 & 335 & 350 & 360 & 375 & 372 & 359 \\
\hline 2 & 95 & 105 & 107 & 89 & 89 & 88 & 100 \\
\hline 3 & 50 & 65 & 56 & 45 & 43 & 46 & 46 \\
\hline 4 & 36 & 45 & 42 & 42 & 43 & 43 & 40 \\
\hline 5 & 15 & 17 & 11 & 19 & 19 & 18 & 17 \\
\hline 6 & 11 & 9 & 14 & 14 & 9 & 9 & 13 \\
\hline 7 & 12 & 14 & 9 & 13 & 12 & 13 & 14 \\
\hline 8 & 8 & 6 & 6 & 6 & 6 & 6 & 6 \\
\hline 9 & 5 & 6 & 5 & 5 & 6 & 6 & 6 \\
\hline 10 & 36 & 31 & 33 & 40 & 31 & 32 & 32 \\
\hline Total & 633 & 633 & 633 & 633 & 633 & 633 & 633 \\
\hline \multicolumn{2}{|c|}{$\begin{array}{l}\text { Degrees of } \\
\text { freedom }\end{array}$} & 6 & 4 & 2 & 6 & 4 & 2 \\
\hline \multicolumn{2}{|c|}{$\begin{array}{l}\text { Estimated values } \\
\text { of parameters }\end{array}$} & $\begin{array}{l}\hat{\alpha}=0.58 \\
\hat{\lambda}_{1}=0.84 \\
\hat{\lambda}_{2}=0.18\end{array}$ & $\begin{array}{l}\hat{\alpha}=0.49 \\
\hat{\lambda}_{1}=0.28 \\
\hat{\lambda}_{2}=0.34 \\
\hat{\rho}_{1}=0.62 \\
\hat{\rho}_{2}=0.52\end{array}$ & $\begin{array}{l}\hat{\alpha}=0.77 \\
\hat{\lambda}_{1}=0.21 \\
\hat{\lambda}_{2}=0.37 \\
\hat{\rho}_{1}=0.56 \\
\hat{\rho}_{2}=0.48 \\
\hat{\mu}_{1}=0.28 \\
\hat{\mu}_{1}=0.36\end{array}$ & $\begin{array}{l}\hat{\alpha}=0.65 \\
\hat{\lambda}_{1}=1.12 \\
\hat{\lambda}_{2}=0.14\end{array}$ & $\begin{array}{l}\hat{\alpha}=0.54 \\
\hat{\lambda}_{1}=0.32 \\
\hat{\lambda}_{2}=0.61 \\
\hat{\rho}_{1}=0.59 \\
\hat{\rho}_{2}=0.84\end{array}$ & $\begin{array}{l}\hat{\alpha}=0.86 \\
\hat{\lambda}_{1}=0.51 \\
\hat{\lambda}_{2}=0.44 \\
\hat{\rho}_{1}=0.54 \\
\hat{\rho}_{2}=0.38 \\
\hat{\mu}_{1}=0.29 \\
\hat{\mu}_{1}=0.35\end{array}$ \\
\hline \multicolumn{2}{|c|}{ Chi-square value } & 11.5 & 7.5 & 3.52 & 5.87 & 4.5 & 2.26 \\
\hline \multicolumn{2}{|c|}{ P-value } & 0.24 & 0.58 & 0.87 & 0.75 & 0.87 & 0.95 \\
\hline
\end{tabular}

Acknowledgements. The authors wish to thank the Editor and the anonymous referee for their fruitful comments on an earlier version of this paper. 


\section{References}

[1] A.C. Cohen, Estimating parameters in a conditional Poisson distribution, Biometrics 16 (1960) 203211.

[2] B.S. Everitt and D.J.Hande, Finite mixture distributions (Chapman and Hall, London, 1981)

[3] M. Huang and K.Y. Fung, (1989), Intervened truncated Poisson distribution. Sankhya Series 51(1989) 302- 310 .

[4] M.G. Kendall, Natural law in science, Journal of Royal Statistical Society, Series A, 124 (1961) 1-18.

[5] C.S. Kumar and D.S. Shibu, Modified intervened Poisson distribution, Statistica 71 (2011) 489-499.

[6] C.S. Kumar and D.S. Shibu, Alternative to truncated intervened Poisson distribution. Journal of Statistics and Applications, 6 (2012) 131-141.

[7] G. McLachlan and D. Peel, Finite mixture models. (Wiley, New York, 2000)

[8] R.Shanmugam, An intervened Poisson distribution and its medical application, Biometrics 41 (1985) 1025-1029.

[9] R. Shanmugam, An inferential procedure for the Poisson intervention parameter, Biometrics 48 (1992) 559- 565.

[10] J. Singh, A characterization of positive Poisson distribution and its application, SIAM Journal of Applied Mathematics 34 (1978) 545-48.

[11] D.M. Titterington, Some recent research in the analysis of mixture distributions, Statistics 21 (1990) 619-641.

[12] D.M. Titterington, A.F.M. Smith, and U.E. Markov (1985). Statistical analysis of finite mixture distributions (Wiley, New York, 1985).

[13] C.B. Williams Number of publications written by biologists, Annals of Eugenics, 12 (1944) $143-146$. 